\title{
Constant Domain 2uantified Modal Logics Without Boolean Negation
}

\author{
Greg Restall* \\ Philosophy Department \\ University of Melbourne \\ restall@unimelb.edu.au
}

Received by Errol Martin and Martin Bunder

Published July 8, 2005

http://philosophy.unimelb.edu.au/ajl/2005

(c) 2005 Greg Restall

\begin{abstract}
This paper provides a sound and complete axiomatisation for constant domain modal logics without Boolean negation. This is a simpler case of the difficult problem of providing a sound and complete axiomatisation for constant-domain quantified relevant logics, which can be seen as a kind of modal logic with a twoplace modal operator, the relevant conditional. The completeness proof is adapted from a proof for classical modal predicate logic (I follow James Garson's presentation of the completeness proof quite closely [II]), but with an important twist, to do with the absence of Boolean negation.
\end{abstract}

There is a natural way to add rules for first-order quantifiers to proof theories for propositional relevant logics, and there is a natural way to add evaluation conditions for quantifiers to the semantics for propositional relevant logics. Kit Fine has shown us that these two natural ways of modelling first order relevant logics do not match up [8]. Furthermore, Fine has given a semantics for

\footnotetext{
${ }^{*}$ I dedicate this paper to Richard Sylvan. He had a deep and abiding interest in relevant logics, including the problem of adding quantifiers in a semantically plausible fashion. His 1980 paper was an important addition to research in the area, but unfortunately, it contains errors which seem insurmountable $[\mathrm{I7}]$. It seems fitting that in an ancestor of this paper I thought I had solved the problems of constant domain quantified relevant logics by proving a stronger version of my Lemma 4 only to find a hole in the 'proof'. I am also indebted to both Bernard Linsky and Ed Zalta $[\overline{I 3}]$ and Max Cresswell [II2] who have, in conversation and in print, convinced me that constant domain semantics are philosophically interesting and defensible. Thanks to an audience at the 1998 Australasian Association for Logic Conference, for helpful discussion on an earlier version of this paper. Comments from an anonymous referee of the fournal, and from Martin Bunder, proved helpful in clarifying the presentation.
} 
the natural proof-theory for quantified relevant logics [7]. Unfortunately, the semantics is quite baroque. This has meant that the semantics has not produced any worthwhile results: The semantics has not been used to prove any interesting metatheoretical results about quantified relevant logics. Furthermore, the semantics has not been given anything like a plausible philosophical justification, or any new understanding of the unique features of quantified relevant logics.

In this paper I will begin to explore the constant domain quantified relevant logics by starting with a simpler case - constant domain modal logics without Boolean negation. That is, I will examine the proof theory and semantics for logics extending distributive lattice logic with extra one place operators. I will provide a sound and complete axiomatisation of the basic normal modal logics extending distributive lattice logic with quantifiers. The completeness proof is adapted from a completeness proof for classical modal predicate logic, but with a twist, seemingly required in the absence of Boolean negation.

These logics are a simplification of relevant logics, which extend distributive lattice logic with the two place intensional connectives of implication and fusion. I end this paper by gesturing towards what needs to be done to extend the results of this paper to the more general setting of relevant logics.

\section{PROPOSITIONAL LOGIC}

I will motivate the propositional fragment of our logic semantically. We are interested in the relation of consequence defined on frames.

DEFINITION I A frame is a partially ordered set $\langle\mathrm{P}, \sqsubseteq\rangle$ equipped with a number of binary relations of accessibility.

The extensional fragment of the language is interpreted in the usual way. We have two propositional constants $T$ and $\perp$, and two binary connectives $\wedge$ and $\checkmark$. The "true at" relation $\Vdash$ between points and propositions is defined recursively. We have a case for atomic propositions which determines the behaviour of the relation $\sqsubseteq$, and the other cases determine the truth of complex propositions in terms of their constituents.

DEFINITION 2 An evaluation relation satisfies the following clauses.

- If $x \sqsubseteq x^{\prime}$ and $x \Vdash p$ then $x^{\prime} \Vdash p$.

- $x \Vdash A \wedge B$ iff $x \Vdash A$ and $x \Vdash B$.

- $x \Vdash A \vee B$ iff $x \Vdash A$ or $x \Vdash B$.

- $x \Vdash T$.

'The expression " $x \Vdash A$ " may be read as " $x$ supports $A$ ", " $x$ forces $A$ ", " $x$ makes $A$ true" or " $A$ is true at $x$ " according to taste. 
- $x \| \perp$.

We do not have Boolean negation for we are interested in ensuring that points can be ordered. Conjunction, disjunction, $\top$ and $\perp$ preserve the hereditary property of evaluations. If $x \Vdash A$ and $x \sqsubseteq x^{\prime}$ then $x^{\prime} \Vdash A$ too. Boolean negation on points (defined by setting $x \Vdash-A$ iff $x \Vdash A$ ) destroys this property. Similarly, the material conditional of classical logic is not allowed, since with $\perp$, it may be used to define Boolean negation. ${ }^{2}$ Distributive lattice logic is no different to the fragment of classical logic without Boolean negation. All of the standard properties in this language (associativity and commutativity of conjunction and disjunction, for example) hold.

This much is straightforward. The logic becomes more interesting when we add operators which exploit the nature of frames. We do this by using binary relations on frames. We allow for four different kinds of modal operators, $\square$ and $\diamond$ are familiar positive operators (called $A$ and I operators) and $\sim$ and $\frown$ are less familiar negative operators (called $E$ and $O$ ) respectively. They are evaluated like this 3

DEFINITION 3 The A, E, I and O operators have the following evaluation conditions:

- $x \Vdash \square A$ iff for all $y$ where $x$ Ry, $y \Vdash A$.

- $x \Vdash \sim A$ iff for no $y$ where $x C y, y \Vdash A$.

- $x \Vdash \diamond A$ iff for some $y$ where $x S y, y \Vdash A$.

- $x \Vdash \frown A$ iff for some $y$ where $x D y, y \Downarrow A$.

In the presence of Boolean negation it suffices to define one of these kinds of operators (say $\square$ ) and to define each of the others in terms of it (as $\diamond$ is of the form $-\square-, \sim$ of the form $\square-$ and $\frown$ of the form $-\square$.) In the absence of Boolean negation this strategy may not work, so we will take all four sorts of operators as primitive.

The hereditary result on formulae works only under special conditions on each accessibility relation. If $x \Vdash \diamond A$ and $x \sqsubseteq x^{\prime}$ then to ensure that $x^{\prime} \Vdash$ $\checkmark A$ we need a condition relating $S$ and $\sqsubseteq$. The requisite conditions for each operator are listed below 4

${ }^{2}$ It follows, then, that in the presentation of the logic of distributive lattices, we need to express it in terms of sequents of the form $A \vdash B$, instead of theorems of the form $\vdash B$. The language has conditional operator with which to convert $A \vdash B$ to $\vdash A \supset B$.

${ }^{3}$ If you are familiar with the theory of the syllogism, you will note why $A, E, I$ and $O$ for the families of one-place connectives. $\square$ is defined by a clause of the form all $\mathrm{A}$ is $\mathrm{B}, \diamond$ by one of the form some $\mathrm{A}$ is $\mathrm{B}, \sim$ by one of the form no $\mathrm{A}$ is $\mathrm{B}$, and $\frown$ by one of the form some $\mathrm{A}$ is not $\mathrm{B}$. These are the four syllogistic forms.

${ }^{4}$ These conditions are discussed in a number of places. The early references are in discussions of intuitionistic modal logic, due to Fischer-Servi [9] and Ewald [6], and discussed at length in Alex Simpson's thesis [19]. They also arise naturally in Dunn's gaggle theory [2, 3]. 
- If $x^{\prime} R y^{\prime}$ and $x \sqsubseteq x^{\prime}$ then there is some $y \sqsubseteq y^{\prime}$ where $x R y$.

- If $x^{\prime} C y$ and $x \sqsubseteq x^{\prime}$ then there is some $y^{\prime} \sqsupseteq y$ where $x C y^{\prime}$.

- If $x \mathrm{Dy} y^{\prime}$ and $x \sqsubseteq x^{\prime}$ then there is some $y \sqsubseteq y^{\prime}$ where $x^{\prime} D y$.

- If $x$ Sy and $x \sqsubseteq x^{\prime}$ then there is some $y^{\prime} \sqsupseteq y$ where $x^{\prime} S y^{\prime}$.

Each of the conditions on accessibility relations connects the accessibility relation with the behaviour of the inclusion relation on the frame. The inclusion relation can be read as the relation in increasing informativeness. If $x \sqsubseteq y$ then all of the information given by $x$ is also given by $y$. Then, thinking of $x \mathrm{Cy}$ as $x$ is compatible with $y$ motivates the condition on $C$. Similarly, reading $x R y$ as those things necessary in $x$ are true in $\mathrm{y}$, and $\mathrm{xSy}$ as $\mathrm{y}$ is possible relative to $\mathrm{x}$, motivates the conditions for $\mathrm{R}$ and $\mathrm{S}$. (The interpretation of $\mathrm{D}$ is left an an exercise for the interested. See An Introduction to Substructural Logics [I6, Chapter II] for more details.)

DEFINITION 4 If $M$ is a model, we say that $A \vdash_{M} B(A$ entails $B$ on $M$ ) just when for each point $x$, if $x \Vdash A$ then $x \Vdash B$. Similarly, if $F$ is a frame, $A \vdash_{F} B$ if and only if $A \vdash_{M} B$ for each model $M$ on $F$. Finally, if $\mathfrak{F}$ is a class of frames, $A \vdash_{\mathfrak{F}} B$ if and only if $A \vdash_{F} B$ for each frame $F \in \mathfrak{F}$.

The interpretation of the extensional connectives $\wedge, \vee, \top, \perp$ ensures that they are standard distributive lattice operators.

DEFINITION 5 A binary relation $\vdash$ on propositions is a distributive lattice relation iff the following conditions hold:

- It is transitive.

- The two-place connective of conjunction is the greatest lower bound for $\vdash$. That is, $C \vdash A \wedge B$ if and only if $C \vdash A$ and $C \vdash B$.

- The two-place connective of disjunction is the least upper bound for $\vdash$. That is, $A \vee B \vdash C$ if and only if $A \vdash C$ and $B \vdash C$.

- Conjunction and disjunction are tied together with the distribution law: $A \wedge(B \vee C) \vdash(A \wedge B) \vee C$.

- There are special propositions $T$ and $\perp$ such that $A \vdash \top$ and $\perp \vdash A$ for each $A$.

Each of the forms of consequence, $\vdash_{M}, \vdash_{F}$ and $\vdash_{\mathfrak{F}}$ are distributive lattice consequence relations. The other modal operators satisfy simple conditions, which we list in another definition.

DEFINITION 6 These are the inference conditions for each kind of operator. 
- The $A$ operator conditions are the inference from $A \vdash B$ to $\square \mathrm{A} \vdash \square \mathrm{B}$, and the entailments $\square A \wedge \square B \vdash \square(A \wedge B)$ and $T \vdash \square T$.

- The $E$ operator conditions are the inference from $A \vdash B$ to $\sim B \vdash \sim A$, and the entailments $\sim A \wedge \sim B \vdash \sim(A \vee B)$ and $T \vdash \sim \perp$.

- The I operator conditions are the inference from $A \vdash B$ to $\diamond A \vdash \diamond B$, and the entailments $\diamond(A \vee B) \vdash \diamond A \vee \diamond B$ and $\diamond \perp \vdash \perp$.

- The $O$ operator conditions are the inference from $A \vdash B$ to $\frown B \vdash \neg A$, and the entailments $\frown(A \vee B) \vdash \frown A \vee \frown B$ and $\frown T \vdash \perp$.

It is not difficult at all to verify that each of these conditions describes the behaviour of the modal operators and the consequence relations $\vdash_{M}, \vdash_{F}$ and $\vdash_{\mathfrak{F}}$. These operators can be defined in pairs in the style of Dunn's Gaggle Theory $[2,3,5]$. For example, $A$ and I operators go together. If $\square$ and $\diamond$ satisfy the two-way rule

$$
\diamond \mathrm{A} \vdash \mathrm{B} \text { if and only if } \mathrm{A} \vdash \square \mathrm{B}
$$

then $\diamond$ is an I operator and $\square$ is an A operator. Similarly, if $\sim$ and $\neg$ satisfy the two-way rule

$$
A \vdash \sim B \text { if and only if } B \vdash \neg A
$$

then they are both $E$ operators. Finally, if $\frown$ and $\smile$ both satisfy the rule

$$
\frown A \vdash B \text { if and only if } \smile B \vdash A
$$

then they are both $\mathrm{O}$ operators. These rules characterise the consequence relation $\vdash_{\mathfrak{F}}$ of the class of all frames. Soundness is trivial to verify. For completeness we can construct a canonical model out of prime theories. If you are interested in the logic of a smaller class of frames, those in which the accessibility relations satisfy interesting properties, you show that the canonical model of such a logic is one of those frames. If you are unlucky and the canonical frame doesn't satisfy the extra conditions, you will need to do more work by constructing a different frame.

This kind of semantic story has very many ancestors. My approach here is most indebted to the work done in the semantics for relevant logics due to Routley and Meyer [18, with further generalisations due to Dunn [2, 4], 5]

\footnotetext{
5The inferences at the heart of this account, connecting $\square$ with $\diamond, \sim$ with $\neg$, and $\frown$ and $\smile$ are more than reminiscent of the display conditions of Nuel Belnap's Display Logic [I]. In each case, we show how to "display" the formula under each operator, in either antecedent or consequent position. However, Belnap's Display Logic is much more than this: it provides an essentially proof-theoretic account of consequence, in which object-level connectives or operators are governed by their connections with other structures in sequents. None of those considerations plays a role here.
} 
We can extend our relation $\vdash$ to relate sets of propositions, by setting $\Sigma \vdash \Delta$ to be true just when some finite conjunction $\bigwedge_{i} A_{i}$ of members of $\Sigma$ entails some disjunction $\bigvee_{i} B_{i}$ of members of $\Delta$. We also use the convention that $\Sigma \vdash \emptyset$ iff $\Sigma \vdash \perp$, and $\vdash \Delta$ iff $\top \vdash \Delta$, and we sometimes write these as ' $\Sigma \vdash$ ', and ' $\vdash \Delta$ ' respectively. (The top and bottom elements $\top$ and $\perp$ are the empty conjunction and disjunction respectively.) By the soundess and completeness results and compactness theorem for these logics, this definition of set-based consequence is equivalent to the obvious frame condition: $\Sigma \vdash_{M} \Delta$ if and only if each point $x$ forcing each element of $\Sigma$ also forces some element of $\Delta$. There is much more that we could do to examine the propositional logics. But for now, we will look at extending the logics to deal with quantifiers.

\section{MODELS FOR QUANTIFIERS}

Our aim is to study the addition of constant domain quantifiers to this breed of model. To add quantifiers to the language, we enrich our language with predicates, constants, variables and the quantifiers $\forall$ and $\exists$. We will define formulae in such a way that we allow formulae with free variables, but we keep sentences to be formulae in which every occuring variable is bound.

For a constant domain model, we have not only a frame, but a non-empty domain of objects $D$. Each n-place predicate $F$ is interpreted by a set $\|F\|_{x}$ of $n$ tuples of D-elements at each point $x$ in the frame. This satisfies the hereditary condition that if $x \sqsubseteq y$ then $\|F\|_{x} \subseteq\|F\|_{y}$.

Terms (either constants or variables) are interpreted by domain elements, independently of the point in the model. (All terms are rigid.) Constants $\mathrm{c}$ are interpreted by objects $\|\mathrm{c}\| \in \mathrm{D}$. Variables $v$ are interpreted by an assignment a which maps variables onto objects. We interpret $v$ by $\|v\|=a(v)$, where $a$ is an assignment. In either case, if $t$ is a term, we use ' $\|t\|_{a}$ ' to name its interpretation, even in the case of constants, where the assignment is irrelevant to the interpretation.

Then we interpret formulas by relativising truth: not only to points in the model, but instead to pairs of points and assignments. Given an assignment a, and a point $x$ we have

$$
\text { - } x, a \Vdash F t_{1} \cdots t_{n} \text { iff }\left\langle\left\|t_{1}\right\|_{a}, \ldots,\left\|v_{n}\right\|_{a}\right\rangle \in\|F\|_{x}
$$

Then to define the quantifiers, we have the standard conditions:

- $x, a \Vdash \forall v A$ iff for each $d \in D, x, a[v:=d] \Vdash A$.

- $x, a \Vdash \exists v A$ iff for some $d \in D, x, a[v:=d] \Vdash A$.

where $a[v:=d]$ is athe $v$-variant of the assignment $a$, which varies from $a$ only by assigning the variable $v$ the value $d$. 
The connectives are interpreted in the usual way, as is the relation of consequence, relative to models, frames and classes of frames. Our job is to investigate these relations of consequence. The first thing to note is that the standard introduction and elimination rules for quantifiers hold.

INTRODUCTION AND ELIMINATION LAWs: For any $A$ with $v$ free we have

$$
\forall v A(v) \vdash_{M} A(c) \text { and } A(c) \vdash_{M} \exists v A(v)
$$

for any model $M$, where $A(c)$ is found by replacing the free occurences of $v$ in $A(v)$ by the name c. These laws follow immediately from the evaluations of $\forall$ and $\exists$. As corollaries of these conditions we have the rules introducing quantifiers as follows:

$$
\frac{\mathrm{A}(\mathrm{c}) \vdash \mathrm{B}}{\forall v \mathrm{~A}(v) \vdash \mathrm{B}}[\forall \text { left }] \quad \frac{\mathrm{B} \vdash \mathrm{A}(\mathrm{c})}{\mathrm{B} \vdash \exists v \mathrm{~A}(v)}[\exists \text { right }]
$$

More interestingly, we have rules for introducing quantifiers on the other side of the turnstile:

$[\forall$ right $]$ If $A \vdash_{F} B(c)$ and $c$ does not occur in $A$ then $A \vdash_{F} \forall v B(v)$.

$[\exists$ left] If $A(c) \vdash B$ and $c$ does not occur in $B$ then $\exists v A(v) \vdash B$.

These hold in any frame $F$. If $A \vdash_{F} B(c)$ then any interpretation in which $x, a \Vdash A$, we must have $x, a \Vdash B(c)$. Now $x, a[v:=d] \Vdash B$ if and only if $x, a \Vdash^{\prime} B(c)$ for the variant interpretation $\Vdash^{\prime}$ which varies from $\Vdash$ only by setting $\|c\|=d$. So, $x, a \Vdash \forall v B(v)$ if and only if $x, a \Vdash^{\prime} B(c)$ for every such variant $\Vdash^{\prime}$. However, $\Vdash^{\prime}$ and $\Vdash$ do not disagree on the formula $A$, as $c$ does not occur in $A$, so if $x, a \Vdash B(c)$ (which we have assumed) we also have $x, a \Vdash^{\prime} B(c)$ too, so we have our result. Similar reasoning verifies the case of the existential quantifier.

These rules ensure that if $v$ is not free in $A$, then $\forall v A$ and $\exists v A$ are equivalent to $A$. For $\forall v A \vdash A$, and since $v$ is not free in $A$, we have $A \vdash A(c)$ for some $c$ not in $A$ (since $A(c)$ is the very same formula as $A$ !) so $A \vdash \forall v A$.

These rules are standard and work for almost any notion of logical consequence with quantifiers. The next results begin to exploit the power of the constant domains.

Distributive Laws: The quantifiers distribute over conjunction and disjunction in the following way.

$$
\forall v(A \vee B) \vdash_{M} \forall v A \vee \exists v B \quad \exists v A \wedge \forall v B \vdash_{M} \exists v(A \wedge B)
$$

These results hold in classical quantificational logic, but the first does not in quantified intuitionistic logic. However, they are straightforward applications of the rules. We will not tarry to verify them here. 
These rules have simpler cases when $B$ does not contain a free $v$. Since in that case $\mathrm{B}$ is equivalent to $\exists v \mathrm{~B}$ and $\forall v \mathrm{~B}$ we have $\forall v(\mathrm{~A} \vee \mathrm{B}) \vdash \forall v \mathrm{~A} \vee \mathrm{B}$, and $\exists v A \wedge B \vdash \exists v(A \wedge B)$.

Intuitionistic logic contains the second of these laws but not the first. Neither of these laws follows from distributive lattice logic together with the introduction and elimination laws ${ }^{6}$

The most interesting results show the interaction between the quantifiers and the extra operators. These results are jointly called the Barcan Laws.

BARCAN LAWS: For any model $M$ and any formula $A$, we have

$$
\begin{array}{ll}
\square \forall v A \dashv \vdash_{M} \forall v \square A & \diamond \exists v A \dashv \vdash_{M} \exists v \diamond A \\
\sim \exists v A \dashv \vdash_{M} \forall v \sim A & \neg \forall v A \dashv \vdash_{M} \exists v \frown A
\end{array}
$$

These results commute a quantifier over a one-place operator. They essentially rely on the fact that the domain $\mathrm{D}$ of quantification stays constant from point to point. They are straightforward to verify. For example, $x, a \Vdash \sim \exists v A$ if and only if for no $y$ where $x C y$ does $y, a \Vdash \exists v A$. That is, iff for no $y$ where $x C y$ does $y, a[v:=d] \Vdash A$ for some $d \in D$, and this just means that for every $\mathrm{d} \in \mathrm{D}$, for no $\mathrm{y}$ does $\mathrm{y}, \mathrm{a}[v:=\mathrm{d}] \Vdash A$. This is equivalent to saying that for every $\mathrm{d} \in \mathrm{D}, \mathrm{x}, \mathrm{a}[v:=\mathrm{d}] \Vdash \sim A$, which is simply $x \Vdash \forall v \sim A$ as desired. If the domain $D$ switched from point to point, we could not reason like this. The other Barcan laws are verified in a similar fashion.

These conditions: the introduction/elimination laws, the distributive laws, and the Barcan laws, jointly give us a good picture the behaviour of the universal and existential quantifiers in constant domain models an frames. It is our job to show that these laws determine the behaviour of the quantifiers on constant domain frames. To do this, however, we need some more tools. Somewhat surprisingly, we need two extra connectives, defined on frames, in order to prove that these rules characterise the logic of constant domain quantification on frames.

\section{NEW CONNECTIVES}

It is well known that you can conservatively add a connective $\supset$ to the language of distributive lattice logic, satisfying the condition

$$
A \wedge B \vdash C \text { if and only if } A \vdash B \supset C
$$

\footnotetext{
${ }^{6}$ For a model in which the introduction and elimination rules hold, use frames with decreasing domains (if $x \sqsubseteq x^{\prime}$ then $\mathrm{D}(x) \supseteq \mathrm{D}\left(x^{\prime}\right)$ ). Set a universally quantified statement to be true at $x$ just when every instance is true at $x$. Existentially quantified sentences are true at $x$ just when some instance is true at some ancestor of $x$. These are the dual conditions to intuitionistic quantifiers, and they verify the distribution of univeral quantifiers over disjunction, but not existential quantifiers over conjunction.
} 
This is intuitionistic implication. (We will call this step $[\wedge$ to $\supset$ ] from left-toright, and $[\supset$ to $\wedge]$ from right to left.) The semantics is reasonably straightforward. We require that frames be extended with a partial order $\sqsubseteq$, and that atomic evaluations be preserved up the order. We set $x \Vdash A \supset B$ iff for each $y$ where $x \sqsubseteq y$, if $y \Vdash A$ then $y \Vdash B$.

Note that with this addition, the unary operator $\neg$ defined by setting as $\neg A$ to $A \supset \perp$ is an $E$ operator. $A \supset \top$ and $\perp \supset A$ are both equivalent to $T$, and $T \supset A$ is equivalent to $A$.

It is less well known that it is just as easy to extend things in the other direction. We can add conservatively add a connective - satisfying the condition

$$
A \vdash B \vee C \text { if and only if } A-B \vdash C
$$

You can read ' $A-B$ ' as ' $A$ without B' 7 The clause is the exact dual of that for $\supset$. (We will call this step $[\vee$ to -$]$ from left-to-right, and $[-$ to $V]$ from right to left.) For each $x, x \Vdash A-B$ iff there is some $y \sqsubseteq x$ where $y \Vdash A$ and $y \Downarrow B$. This preserves the hereditary condition, and so, is a conservative extension of intuitionistic propositional logic and therefore, of distributive lattice logic 8 The addition of subtraction and implication means that we can work "under" conjunction in antecedent position and disjunction in consequent position. Any fact of the form $C \wedge A \vdash B \vee D$ may be transformed into $(C \wedge A)-D \vdash B$ (which may prove useful, if $B$ has a special property, such as having a variable free not present in the other formulas) or into $A \vdash C \supset(B \vee D)$ (if we wish to isolate $A$ ). These properties will play a role in the derivation of crucial lemmas in the completeness proof in the next section.

Note too that $\frown$, defined by setting $\frown A$ as $T-A$ is an $O$ operator. $\perp-A$ and $A-T$ are both equivalent to $\perp$, while $A-\perp$ is equivalent to $A$.

You get conditions tying quantifiers to the conditional and to subtraction.

$$
\forall v(\mathrm{~A} \supset \mathrm{B}) \vdash_{M} \forall v \mathrm{~A} \supset \forall v \mathrm{~B} \quad \exists v \mathrm{~B}-\exists v \mathrm{~A} \vdash_{M} \exists v(\mathrm{~B}-\mathrm{A})
$$

These can be verified semantically in a straightforward fashion. More interesting is the fact that they follow given the rules of proof already at hand. We start with the subtraction case as it is less familiar. The proof uses the rules we have introduced, governing subtraction, and the existential quantifier. The only other principle implicitly appealed to is the commutativity of disjunction. We use the principle that from $B-A \vdash C$ we can infer $B \vdash A \vee C$, and then, $B \vdash C \vee A$ and to return, $B-C \vdash A$. Instead of making the commutation step of the disjunction explicit, we present such a sequence of inferences as leading from $B-A \vdash C$ to $B \vdash A \vee C$, using $[-$ to $V]$ and then we pull the other

\footnotetext{
${ }^{7}$ Subtraction is regularly rediscovered in the literature. The first reference to it of which I am aware is in the work of Rauszer [14, [5].

${ }^{8}$ It is not a conservative extension to intuitionistic predicate logic, however. As we shall see, with subtraction, we can prove $\forall x(A \vee B) \vdash \forall x A \vee \exists v B$
} 
disjunct of the disjunction back to form $B-C \vdash A$, using $[V$ to -$]$.

$$
\begin{gathered}
\frac{B(c)-A(c) \vdash B(c)-A(c)}{B(c) \vdash A(c) \vee(B(c)-A(c))}[- \text { to } \vee] \\
\frac{B(c)-(B(c)-A(c)) \vdash A(c)}{B(c)-(B(c)-A(c)) \vdash \exists v A}[\exists \text { right }] \\
\frac{B(c) \vdash \exists v A \vee(B(c)-A(c))}{B(- \text { to } \vee]} \\
\frac{B(c) \vdash \exists v A \vee \exists v(B-A)}{\exists v B \vdash \exists v A \vee \exists v(B-A)}[\exists \text { left }] \\
\frac{\exists v \text { right }]}{\exists v B-\exists v A \vdash \exists v(B-A)}[\vee \text { to }-]
\end{gathered}
$$

Furthermore, we have the "flipping" rules

$$
\forall v(\mathrm{~A} \supset \mathrm{B}) \vdash \exists v \mathrm{~A} \supset \mathrm{B} \quad \mathrm{B}-\forall v \mathrm{~A} \vdash \exists v(\mathrm{~B}-\mathrm{A})
$$

where $v$ is not free in B. These are verified similarly. Here is the subtraction case.

$$
\begin{aligned}
& \frac{B-A(c) \vdash B-A(c)}{B-A(c) \vdash \exists v(B-A)}[\exists \text { right }] \\
& \frac{B \vdash A(c) \vee \exists v(B-A)}{B-\text { to } V]}[\vee \text { to }-] \\
& \frac{B-\exists v(B-A) \vdash A(c)}{B-\exists v(B-A) \vdash \forall v A}[\forall \text { right }] \\
& \frac{B \vdash \forall v A \vee \exists v(B-A)}{B-\forall v A \vdash \exists v(B-A)}[- \text { to } V]
\end{aligned}
$$

Finally, and most interestingly, the introduction and elimination laws, together with implication and subtraction, is enough to verify the distribution laws.

$$
\begin{aligned}
& \frac{A(c) \vee B(c) \vdash A(c) \vee B(c)}{\forall v(A \vee B) \vdash A(c) \vee B(c)}[\forall \text { left }] \\
& \frac{\forall v(A \vee B)-A(c) \vdash B(c)}{\forall}[\text { to }-] \\
& \frac{\forall v(A \vee B)-A(c) \vdash \exists v B}{\forall v(A \vee B g h t]}[- \text { to } \vee] \\
& \frac{\forall v(A \vee B)-\exists v B \vdash A(c)}{\forall \vee ~}[\text { to }-] \\
& \frac{\forall v(A \vee B)-\exists v B \vdash \forall v A}{\forall v(A \vee B) \vdash \forall v A \vee \exists v B}[- \text { to } \vee]
\end{aligned}
$$

These connectives are definable on any constant domain frame using the structure already existing on that frame. Much more can be said about them (especially subtraction, which is much less studied than implication). However, all 
of this is preliminary to our present project of adding constant domain quantifiers to our logic. It will become clear why having implication and subtraction at our disposal helps when we get to the completeness proof.

\section{COMPLETENESS}

For completeness we will construct a canonical frame and model. We will show that if $\Sigma \nvdash \Delta$ (in the proof theory) then there is a point in the canonical model in which every element of $\Sigma$ is true but every element of $\Delta$ is false. The completeness proof is not too different from that in either classical modal logic or that in propositional relevant logics. It is handy to define pairs of sets of formulae. The left member of a pair is the set of formulae we wish to make true and the right member of the pair is the set of formulae we wish to see fail. The proof here is a fairly straightforward adaptation of the classical result (I follow reasonably closely the presentation of Garson [IO]], with the operators of intuitionistic implication and subtraction doing their duty in the absence of Boolean negation).

DEFINITION 7 The pair $\langle\Sigma, \Delta\rangle$ is said to be a $\vdash$-pair (or simply, a pair in what follows) if $\Sigma \not \forall \Delta$. A pair $\langle\Sigma, \Delta\rangle$ is quantifier suited iff the following two conditions hold:

- If $\Sigma \vdash \Delta \cup\{A(\mathrm{c})\}$ for each $\mathrm{c}$ then $\Sigma \vdash \Delta \cup\{\forall v A\}$ for each $v$.

- If $\Sigma \cup\{A(c)\} \vdash \Delta$ for each $c$ then $\Sigma \cup\{\exists v A\} \vdash \Delta$ for each $v$.

A pair $\langle\Sigma, \Delta\rangle$ is said to be full in a language $\mathrm{L}$ iff $\Sigma \cup \Delta=\mathrm{L}$.

Our canonical frame will be constructed from full quantifier-suited pairs from a particular language. The left set in such a pair is a good match for a point in a model. The next result is simple to prove.

LEMMA I If $\langle\Sigma, \Delta\rangle$ is a full pair, it followes that $\Sigma$ is a prime theory. If $\langle\Sigma, \Delta\rangle$ is full and quantifier-suited then $\exists \nu A \in \Sigma$ if and only if $\mathrm{A}(\mathrm{c}) \in \Sigma$ for some name $\mathrm{c}$, and $\forall v A \in \Sigma$ if and only if $\mathrm{A}(\mathrm{c}) \in \Sigma$ for all names $\mathrm{c}$.

A prime theory satisfies each of the required distributive lattice conditions. If $\Sigma$ is a prime theory then $T \in \Sigma, \perp \notin \Sigma, A \wedge B \in \Sigma$ if and only if $A, B \in \Sigma$, and $A \vee B \in \Sigma$ if and only if either $A$ or $B$ is in $\Sigma$. If the pair is also quantifier-suited then the quantifiers are interpreted in the desired way too. $\forall v A \in \Sigma$ if and only if $A$ (c) $\in \Sigma$ for each name c. $\exists v A \in \Sigma$ if and only if $A(c) \in \Sigma$ for some name c.

Now we show how toconstruct full quantifier-suited pairs. Firstly, we have the technique needed to get one in the first place.

LEMMA 2 (PAIR EXTENSION LEMMA I) If $\langle\Sigma, \Delta\rangle$ is a pair then there is also a full quantifier-suited pair $\left\langle\Sigma^{\prime}, \Delta^{\prime}\right\rangle$ extending $\langle\Sigma, \Delta\rangle$, in a new language extending the original language by at most countably many new constants. 
Proof: This is achieved by enumerating the formulae in the language $A_{0}, A_{1}$, ... and defining the pair $\left\langle\Sigma_{0}, \Delta_{0}\right\rangle$ to be $\langle\Sigma, \Delta\rangle$, and $\left\langle\Sigma_{n+1}, \Delta_{n+1}\right\rangle$ is defined as follows:

- If $\Sigma_{n} \cup\left\{A_{n}\right\} \not \forall \Delta_{n}$ and $A_{n}$ is not of the form $\exists v B$ then $\left\langle\Sigma_{n+1}, \Delta_{n+1}\right\rangle=$ $\left\langle\Sigma_{n} \cup\left\{A_{n}\right\}, \Delta_{n}\right\rangle$.

- If $\Sigma_{n} \cup\left\{A_{n}\right\} \forall \Delta_{n}$ and $A_{n}$ is of the form $\exists v B$ then $\left\langle\Sigma_{n+1}, \Delta_{n+1}\right\rangle=$ $\left\langle\Sigma_{n} \cup\left\{A_{n}, B(c)\right\}, \Delta_{n}\right\rangle$, where $c$ is a new constant not appearing in $\Sigma_{n}$, $A_{n}$ or $\Delta_{n}$.

- If $\Sigma_{n} \cup\left\{A_{n}\right\} \vdash \Delta_{n}$ and $A_{n}$ is not of the form $\forall v B$ then $\left\langle\Sigma_{n+1}, \Delta_{n+1}\right\rangle=$ $\left\langle\Sigma_{n}, \Delta_{n} \cup\left\{A_{n}\right\}\right\rangle$.

- If $\Sigma_{n} \cup\left\{A_{n}\right\} \vdash \Delta_{n}$ and $A_{n}$ is of the form $\forall v B$ then $\left\langle\Sigma_{n+1}, \Delta_{n+1}\right\rangle=$ $\left\langle\Sigma_{n}, \Delta_{n} \cup\left\{A_{n}, B(c)\right\}\right\rangle$, where $c$ is a new constant not appearing in $\Sigma_{n}$, $A_{n}$ or $\Delta_{n}$.

Then we set $\left\langle\Sigma^{\prime}, \Delta^{\prime}\right\rangle$ to be $\left\langle\bigcup_{n} \Sigma_{n}, \bigcup_{n} \Delta_{n}\right\rangle$. This is a partition of the formulas by construction: every formula $A_{n}$ is either in $\Sigma^{\prime}$ or $\Delta^{\prime}$.

We next show that it is a pair: We show that $\Sigma^{\prime} \not \Delta^{\prime}$. To show this, given the compactness of $\vdash$ it suffices to show that $\Sigma_{n} \not \Delta_{n}$ for each $n$. We show this by induction on $n$. The result holds for $n=0$ by hypothesis. For $n+1$, suppose $\Sigma_{n+1} \vdash \Delta_{n+1}$ but that $\Sigma_{n} \not \Delta_{n}$. It follows that we cannot be in the first case of the construction, for that assurs $\Sigma_{n+1} \not \forall \Delta_{n+1}$ explicitly. Suppose $A_{n}$ is of the form $\forall v B$, and that while $\Sigma_{n} \cup\left\{A_{n}\right\} \forall \Delta_{n}$ we have $\Sigma_{n} \cup\left\{A_{n}, B(c)\right\} \vdash \Delta_{n}$ for a $c$ new to $\Sigma_{n}$ and $\Delta_{n}$ and $A_{n}$. Therefore there is a conjunction $C$ of members of $\Sigma_{n}$, and a disjunction $D$ from $\Delta_{n}$ such that $C \wedge A_{n} \wedge B(c) \vdash D$. It follows that $\exists v\left(C \wedge A_{n} \wedge B\right) \vdash D$ by existential introduction. By the existential distribution fact we have $C \wedge A_{n} \wedge \exists v B \vdash D$ ( $c$ is new to $C, A_{n}$ and $D$ ) and hence $C \wedge A_{n} \vdash D$, contrary to our hypothesis that $\Sigma_{n} \cup\left\{A_{n}\right\} \forall \Delta_{n}$. So, this case assures us that $\Sigma_{n+1} \not \forall \Delta_{n+1}$.

Now suppose we are in the third case, and that both $\Sigma_{n} \cup\left\{A_{n}\right\} \vdash \Delta_{n}$ and that $\Sigma_{n} \vdash \Delta_{n} \cup\left\{A_{n}\right\}$. Then for some $C \in \bigwedge \Sigma_{n}$ and $D \in \bigvee \Delta_{n}$ we have $C \wedge A_{n} \vdash D$ and $C \vdash A_{n} \vee D$. Disjoining $D$ to the first, we have $\left(C \wedge A_{n}\right) \vdash D$, and conjoining $C$ to the second, we have $C \vdash C \wedge\left(A_{n} \vee D\right)$. Distribution gives us $C \vdash D$, which we already know not to be the case, since $\Sigma_{n} \forall \Delta_{n}$ by hypothesis. So in this case too, $\Sigma_{n+1} \not \forall \Delta_{n+1}$.

The final case is similar, except that we know that $A_{n}$ has the form $\exists v B$, and we have $C \wedge A_{n} \vdash D$ and $C \vdash A_{n} \vee B(c) \vee D$. Conjoining $C$ we get $C \vdash C \wedge\left(A_{n} \vee B(c) \vee D\right)$ for each $c$ (and in particular, a $c$ absent from $C, A_{n}$ and $D)$ and hence $C \vdash \forall v\left(C \wedge\left(A_{n} \vee B \vee D\right)\right) \vdash C \wedge\left(A_{n} \vee \forall v B \vee D\right)=C \wedge\left(A_{n} \vee D\right)$, and we proceed as before to get $C \vdash D$, which contradicts the assumption, giving then $\Sigma_{n+1} \not \forall \Delta_{n+1}$. 
The pair $\left\langle\Sigma^{\prime}, \Delta^{\prime}\right\rangle$ is quantifier suited by construction too. If $\Sigma^{\prime} \vdash \Delta^{\prime} \cup$ $\{A(c)\}$ for each constant $c$ then we must have $A(c) \in \Sigma^{\prime}$ for each $c$, and hence $\forall v A \in \Sigma^{\prime}$, giving $\Sigma^{\prime} \vdash \Delta^{\prime} \cup\{\forall v A\}$. The existential quantifier case is dual. $\sharp$ Now once we have a domain, we want to keep it. This is where the rather weak definition of quantifier-suitedness comes in.

LEMMA 3 (PAI R EXTENSION LEMMA 2) If $\langle\Sigma, \Delta\rangle$ is a quantifier-suited pair, and if one of $\Sigma$ and $\Delta$ is finite, then there is a full quantifier-suited pair $\left\langle\Sigma^{\prime}, \Delta^{\prime}\right\rangle$ extending $\langle\Sigma, \Delta\rangle$, in the same language.

To prove this lemma we need the following simple lemma.

LEMMA 4 (FINITE ADDiTION LEMMA) If $\langle\Sigma, \Delta\rangle$ is a quantifier-suited pair and $\mathrm{X}$ and $\mathrm{Y}$ are finite sets of formulas in the same language, and one of $\Sigma$ and $\Delta i$ is finite, then $\langle\Sigma \cup X, \Delta \cup Y\rangle$ is also quantifier-suited.

This is the result for which we need subtraction and intuitionistic implication.

Proof: Here is the case where $\Sigma$ is finite. By abuse of notation, I will let " $X$ " and "Y" stand for the formulae $\bigwedge X$ and $\bigvee Y$. Let $B$ be the formula $\bigwedge \Sigma$.

Now, if $\Sigma \cup X \cup\{A(c)\} \vdash \Delta \cup Y$ for each name c, we have $B \wedge X \wedge A(c) \vdash \Delta \cup Y$, and equivalently, $(B \wedge X \wedge A(c))-Y \vdash \Delta$. So, by the quantifier-suitedness of $\langle\Sigma, \Delta\rangle$ we have $(\exists v)((\mathrm{B} \wedge \mathrm{X} \wedge \mathrm{A})-\mathrm{Y}) \vdash \Delta$. As a result, $(\mathrm{B} \wedge \mathrm{X} \wedge \exists v \mathrm{~A})-\mathrm{Y} \vdash \Delta$, and hence $\Sigma \cup X \cup\{\exists v A\} \vdash \Delta \cup Y$ as desired.

If $\Sigma \cup X \vdash \Delta \cup Y \cup\{A(c)\}$ for each name $c$, we have $(B \wedge X)-(Y \vee A(c)) \vdash \Delta$ for each $\mathrm{c}$, and hence $\exists v((\mathrm{~B} \wedge \mathrm{X})-(\mathrm{Y} \vee A)) \vdash \Delta$. But this gives $(\mathrm{B} \wedge \mathrm{X})-(\mathrm{Y} \vee \forall \vee A) \vdash$ $\Delta$ and hence $\Sigma \cup X \vdash \Delta \cup Y \cup\{\forall v A\}$ as desired.

The case for finite $\Delta$ is dual, using implication instead of subtraction. $\quad \sharp$ Given the finite addition lemma, we can prove the second pair extension lemma. Proof: The process is similar to that used in the proof of the first pair extension lemma. Now, however, instead of adding a new witness for each existential quantifier, we show that an old one will do.

- If $\Sigma_{n} \cup\left\{A_{n}\right\} \not \forall \Delta_{n}$ and $A_{n}$ is not of the form $\exists v B$, then $\left\langle\Sigma_{n+1}, \Delta_{n+1}\right\rangle=$ $\left\langle\Sigma_{n} \cup\left\{A_{n}\right\}, \Delta_{n}\right\rangle$.

- If $\Sigma_{n} \cup\left\{A_{n}\right\} \forall \Delta_{n}$ and $A_{n}$ is of the form $\exists v B$, then $\left\langle\Sigma_{n+1}, \Delta_{n+1}\right\rangle=$ $\left\langle\Sigma_{n} \cup\left\{A_{n}, B(c)\right\}, \Delta_{n}\right\rangle$, for some constant $c$ where $\Sigma_{n} \cup\left\{A_{n}, B[v:=c]\right\} \forall$ $\Delta_{n}$.

- If $\Sigma_{n} \cup\left\{A_{n}\right\} \vdash \Delta_{n}$ and $A_{n}$ is not of the form $\forall v B$, then $\left\langle\Sigma_{n+1}, \Delta_{n+1}\right\rangle=$ $\left\langle\Sigma_{n}, \Delta_{n} \cup\left\{A_{n}\right\}\right\rangle$.

- If $\Sigma_{n} \cup\left\{A_{n}\right\} \vdash \Delta_{n}$ and $A_{n}$ is of the form $\forall v B$, then $\left\langle\Sigma_{n+1}, \Delta_{n+1}\right\rangle=$ $\left\langle\Sigma_{n}, \Delta_{n} \cup\left\{A_{n}, B(c)\right\}\right\rangle$, for some constant $c$ where $\Sigma_{n} \not \forall \Delta_{n} \cup\left\{A_{n}, B(c)\right\}$. 
We need show that at each stage $n$, if we use the second or fourth lines of the definition, an appropraite $\mathrm{c}$ can be found. For this, we appeal to the finite addition lemma: Each $\left\langle\Sigma_{n}, \Delta_{n}\right\rangle$ is quantifier-suited, as it is a finite extension to the quantifier-suited $\langle\Sigma, \Delta\rangle$, and one of $\Sigma$ and $\Delta$ is finite.

Now, suppose we are in the second case. If there is no suitable $c$ such that $\Sigma_{n} \cup\left\{A_{n}, B[v:=c]\right\} \not \Delta_{n}$. It follows that for each c, $\Sigma_{n} \cup\left\{A_{n}, B(c)\right\} \vdash \Delta_{n}$. Therefore since $\Sigma_{n}$ is quantifier suited, $\Sigma_{n} \cup\left\{A_{n}\right\} \vdash \Delta_{n}$. However, this is a contradiction. The fourth case is dual.

Now we must construct theories to use in the canonical model. The strategy is simple. If $\Sigma \forall \Delta$, we extend $\langle\Sigma, \Delta\rangle$ to a full quantifier-suited $\left\langle\Sigma^{\prime}, \Delta^{\prime}\right\rangle$. Then we use the class of full quantifier-suited pairs in this language as our canonical frame. Since they are full and quantifier-suited we know that these points interpret the extensional part of the language adequately. We must ensure that we interpret the modal operators well too. We define the accessibility relations in the obvious way.

- $\Sigma R \Gamma$ if and only if for each $A$, if $\square A \in \Sigma$ then $A \in \Gamma$.

- $\Sigma S \Gamma$ if and only if for each $A$, if $A \in \Gamma$ then $\diamond A \in \Sigma$.

- $\Sigma C \Gamma$ if and only if for each $A$, if $\sim A \in \Sigma$, then $A \notin \Gamma$.

- $\Sigma D \Delta$ if and only if for each $A$, if $A \notin \Gamma$, then $\frown A \in \Sigma$.

These conditions ensure that the accessibility relations satisfy the required interactions with the ordering on the canonical frame. This order is the subsethood relation on the theories.

These definitions of accessibility relations immediately ensure half of the evaluation conditions for modal operators. If $\square A \in \Sigma$ then for each $\Gamma$ where $\Sigma R \Gamma, A \in \Gamma$. To complete the picture we wish to show that if $\square A \notin \Sigma$ then there is some $\Gamma$ where $\Sigma R \Gamma$ and $A \notin \Gamma$. This is slightly more difficult than in the propositional case, for we need to not only construct the theory, but also ensure that it is quantifier-suited in the same language. To do this we use the following lemma. This is the point at which the Barcan Laws are used.

LEMMA 5 If $\langle\Sigma, \Delta\rangle$ is full and quantifier suited, then

- If $\square \mathrm{B} \notin \Sigma$ then $\left\langle\square^{-1} \Sigma,\{\mathrm{B}\}\right\rangle$ is a quantifier-suited pair.

- If $\neg \mathrm{B} \notin \Sigma$ then $\left\langle\{\mathrm{B}\}, \neg^{-1} \Sigma\right\rangle$ is a quantifier-suited pair.

- If $\diamond \mathrm{B} \in \Sigma$, then $\left\langle\{\mathrm{B}\}, \diamond^{-1} \Delta\right\rangle$ is a quantifier-suited pair.

- If $\frown \mathrm{B} \in \Sigma$, then $\left\langle\frown^{-1} \Delta,\{\mathrm{B}\}\right\rangle$ is a quantifier-suited pair.

- If $\mathrm{A} \supset \mathrm{B} \notin \Sigma$, then $\langle\Sigma \cup\{\mathrm{A}\},\{\mathrm{B}\}\rangle$ is a quantifier-suited pair.

- If $\mathrm{A}-\mathrm{B} \in \Sigma$, then $\langle\{\mathrm{A}\},\{\mathrm{B}\} \cup \Delta\rangle$ is a quantifier-suited pair. 
Proof: Let's look at three cases: for $\square, \frown$ and subtraction. First, the $\square$ case: $\left\langle\square^{-1} \Sigma,\{B\}\right\rangle$ is a pair iff $\bigwedge_{i} A_{i} \forall B$ for any finite set of $A_{i} s$ in $\square^{-1} \Sigma$. But this is simple: if $\bigwedge_{i} A_{i} \vdash B$, then $\square \bigwedge_{i} A_{i} \vdash \square B$, and hence $\bigwedge_{i} \square A_{i} \vdash \square B$, giving $\square \mathrm{B} \in \Sigma$ (since each $\square A_{i} \in \Sigma$ ) contrary to our assumption.

By the finite addition lemma, $\left\langle\square^{-1} \Sigma,\{B\}\right\rangle$ is quantifier suited if $\left\langle\square^{-1} \Sigma, \emptyset\right\rangle$ is. And for this we first need to show that if $\square^{-1} \Sigma \vdash A(c)$ for each $c$ then $\square^{-1} \Sigma \vdash \forall v A$. So, if $\bigwedge_{i} A_{i} \vdash A$ (c) for each c, it follows that $\square \bigwedge_{i} A_{i} \vdash \square A$ (c) for each $v$, and hence, $\bigwedge_{i} \square A_{i} \vdash \square A$ (c), giving $\Sigma \vdash A$ (c) for each c. Since $\langle\Sigma, \Delta\rangle$ is quantifier suited, it follows that $\forall v \square A \in \Sigma$. The Barcan formula then gives $\square \forall v A \in \Sigma$. As a result, $\square^{-1} \Sigma \vdash \forall v A$ as desired.

For the second half, it is sufficient to show that if $\square^{-1} \Sigma \cup\{A(c)\} \vdash \perp$ for each $\mathrm{c}$ then $\square^{-1} \Sigma \vdash A(\mathrm{c}) \supset \perp$ for each $\mathrm{c}$, so the previous result applies, giving us $\square^{-1} \Sigma \vdash \forall v(A \supset \perp)$ and hence $\square^{-1} \Sigma \vdash \exists v A \supset \perp$ giving $\square^{-1} \Sigma \cup\{\exists v A\} \vdash \perp$ as desired.

Now for the $\frown$ case. First we show that $\left\langle\frown^{-1} \Delta,\{B\}\right\rangle$ is a pair. If $\bigwedge_{i} A_{i} \vdash B$ where $A_{i} \in \frown^{-1} \Delta$, then by the $O$ conditions, $\frown B \vdash \frown \bigwedge_{i} A_{i}$ but $\frown \bigwedge_{i} A_{i} \vdash$ $\bigvee_{i} \frown A_{i}$. But $\frown B \in \Sigma$, and this gives $\Sigma \vdash \Delta$ contrary to our assumption. So $\left\langle\frown^{-1} \Delta,\{B\}\right\rangle$ is a pair.

To show that it is quantifier suited, it suffices to show that $\left\langle\frown^{-1} \Delta, \emptyset\right\rangle$ is quantifier suited. For this, suppose $\frown^{-1} \Delta \vdash \mathrm{C}$ (c) for each c. We then have $\frown C(c) \vdash \frown \bigwedge_{i} A_{i}$ and hence $\frown C(c) \vdash \bigvee_{i} \frown A_{i}$ (for a choice of the $A_{i}$ from $\left.\frown^{-1} \Delta\right)$. So, $\neg C(\mathrm{c}) \vdash \Delta$ and by the quantifier suitedness of the original pair, $\exists v \frown C \vdash \Delta$. By the Barcan Law for $\frown$ we get $\frown \forall v C \vdash \Delta$, and hence $\frown^{-1} \Delta \vdash$ $\forall v \mathrm{C}$ as desired.

For the second half, it is sufficient to show that if $\frown^{-1} \Delta \cup\{A(c)\} \vdash \perp$ for each $\mathrm{c}$ then $\frown^{-1} \Delta \vdash A(\mathrm{c}) \supset \perp$ for each $\mathrm{c}$ and as before, the previous result applies, giving us $\frown^{-1} \Delta \vdash \forall v(A \supset \perp)$ and hence $\frown^{-1} \Delta \vdash \exists v A \supset \perp$ giving $\frown^{-1} \Delta \cup\{\exists v A\} \vdash \perp$ as desired.

Finally, for subtraction, we wish to show that $\langle\{A\},\{B\} \cup \Delta\rangle$ is a pair if $A-B \notin \Sigma$. This is immediate, for if $A \vdash\{B\} \cup \Delta$, then $A-B \vdash \Delta$. To show that it is quantifier suited, we wish to show that $\langle\emptyset,\{B\} \cup \Delta\rangle$ is quantifier suited. For this, if $C(c) \vdash\{B\} \cup \Delta$ for each $c$, we have $C(c)-B \vdash \Delta$ for each $c$ and by the quantifier suitedness of the original pair, $\exists v(C-B) \vdash \Delta$ and hence $\exists v \mathrm{C}-\mathrm{B} \vdash \Delta$ (the $v$ can be chosen to be absent from $\mathrm{B}$ ) giving $\exists v \mathrm{C} \vdash \Delta \cup\{\mathrm{B}\}$. Furthermore, if $T \vdash\{C(c), B\} \cup \Delta$ for each $c$, we have $T-C(c) \vdash\{B\} \cup \Delta$ for each c, giving us $\exists v(T-C) \vdash\{B\} \cup \Delta$ and hence $T \vdash\{\forall v C, B\} \cup \Delta$ as desired. $\sharp$ These lemmas jointly give us our completeness proof.

THEOREM 6 (COMPLETENess) If $\Sigma \forall \forall \Delta$ then there is some model $M$ in which $\Sigma \nvdash{ }_{M} \Delta$.

Proof: Extend $\langle\Sigma, \Delta\rangle$ to a full quantifier suited pair $\left\langle\Sigma^{\prime}, \Delta^{\prime}\right\rangle$ by the first pair extension lemma. Construct a frame on the set of all full quantifier suited pairs on the language of $\left\langle\Sigma^{\prime}, \Delta^{\prime}\right\rangle$. The domain of this frame is the set of constants 
in the language. The extension of a predicate $F$ at the a point $\langle\Gamma, \Pi\rangle$ is the set of $n$-tuples $\left\langle c_{1}, \ldots, c_{n}\right\rangle$ such that $F_{1} \cdots c_{n} \in \Gamma$. The lemmas proved above ensure that all of the connective evaluation conditions hold in the canonical frame, such that $\langle\Gamma, \Pi\rangle \Vdash A$ if and only if $A \in \Gamma$.

\section{MISCELLANY}

What of THE NEW CONnectives? Much more should be said about the new connectives $\supset$ and - . Can the finite addition lemma be proved without them? This would certianly be an advantage. Are these connectives meaningful in any logics for which we want constant domain frames?

RESTRICTED QUANTIFICATION We can let E! be an existence predicate, and define $\left(\forall_{E !} x\right) A$ to be $(\forall x)(E ! x \supset A)$, and $\left(\exists_{E !} x\right) A$ to be $(\exists x)(E ! x \wedge A)$. This mimics increasing domain logics in a familiar fashion.

More oddly, we can define $\mathrm{N}$ ! as a nonexistence predicate, to define $\left(\forall_{\mathrm{N}} ! \mathrm{X}\right) \mathrm{A}$ as $(\forall x)(N ! x \vee A)$, and $\left(\exists_{N !} x\right)$ to be $(\exists x)(A-N ! x)$. This mimics decreasing domain logics. Is there any use for this dualisation of the intuitionistic case?

What about LOgics without Distribution? None of this works in the absence of distribution of conjunction over disjunction. For an analagous semantics to the frames presented here, you should examine the linear logic literature on phase spaces [II, [6].

DUNN CONDITIONS An obvious pair of conditions to want $\square$ and $\diamond$ to satisfy is what I call the Dunn Conditions [5]:

$$
\square(\mathrm{A} \vee \mathrm{B}) \vdash \square \mathrm{A} \vee \diamond \mathrm{B} \quad \diamond \mathrm{A} \wedge \square \mathrm{B} \vdash \diamond(\mathrm{A} \wedge \mathrm{B})
$$

(They should look reminisicent of the quantifier distribution laws.) These are valid if $\square$ and $\diamond$ use the same accessibility relation. To prove the addition sound and complete for the obvious class of frames we need a stronger version of the finite addition lemma. For given $\square A \notin \Sigma$ (with a quantifier suited pair $\langle\Sigma, \Delta\rangle$ ) we need $\left\langle\square^{-1} \Sigma,\{A\} \cup \nabla^{-1} \Delta\right\rangle$ to be quantifier suited. At the new accessible point, everything necessary at $\Sigma$ is true, and everything not possible at $\Sigma$ is not true. The current proof of the finite addition lemma requires that one of the parts of the pair be finite. This does not hold here. To prove completeness we need a stronger version of the finite addition lemma or a completely different proof.

ImplicATion AND Fusion Implication and fusion seem to cause more difficulties too. For example, with implication, if $A \rightarrow B \notin \Sigma$ where $\langle\Sigma, \Delta\rangle$ is quantifier-suited, we need to construct two pairs, one at which $A$ holds (that is simple: show that $\langle\{A\},\{C: C \rightarrow B \in \Sigma\}\rangle$ is quantifier-suited and then extend that to a full quantifier suited pair $\langle\Gamma, \Pi\rangle)$ and another at which $B$ fails. This one 
is more difficult - we wish to show that $\langle\Sigma \Gamma,\{B\}\rangle$ is quantifier-suited, where $\Sigma \Gamma=\{\mathrm{C} \circ \mathrm{D}: \mathrm{C} \in \Sigma, \mathrm{D} \in \Gamma\}$. How do you do this?

IDENTITY There is no doubt that there are choices to be made in the semantics of identity. If the extension of the identity predicate is reflexive at all points, then we have $A \vdash a=a$ for any proposition $A$. This will be anathema to relevant logicians. To give purchase to a relevant account of identity, we must have points at which identity fails to be reflexive. The validity of $\mathrm{a}=\mathrm{b} \vdash \mathrm{b}=\mathrm{a}$ ensures that identity is symmetric at all points. If $\mathrm{a}=\mathrm{b} \wedge \mathrm{b}=\mathrm{c} \vdash \mathrm{a}=\mathrm{c}$, then identity must be transitive at all points.

\section{REFERENCES}

[I] NUel D. Belnap. "Display Logic". Fournal of Philosophical Logic, II:375-417, 1982.

[2] J. Michael DunN. "Gaggle Theory: An Abstraction of Galois Connections and Residuation with Applications to Negation and Various Logical Operations". In Logics in AI, Proceedings European Workshop FELIA 1990, volume 478 of Lecture Notes in Computer Science. Springer-Verlag, I99I.

[3] J. michael dunn. "Gaggle Theory Applied to Modal, Intuitionistic and Relevance Logics". In I. MAX AND w. STELZNER, editors, Logik und Mathematik: Frege-Kolloquium fena. de Gruyter, 1993.

[4] J. Michael Dunn. "Star and Perp: Two Treatments of Negation". In James e. tomber lin, editor, Philosophical Perspectives, volume 7, pages 33I-357. Ridgeview Publishing Company, Atascadero, California, 1994.

[5] J.michael dunn. "Positive Modal Logic". Studia Logica, 55:30I-317, 1995.

[6] william B. EWald. "Intuitionistic Tense and Modal Logic". Fournal of Symbolic Logic, 51:166-179, 1986.

[7] Kit Fine. "Semantics for Quantified Relevance Logic". Fournal of Philosophical Logic, $17: 27-59,1988$.

[8] Kit Fine. "Incompleteness for Quantified Relevance Logics". In JeAn NORMan AND RICHARD sylvan, editors, Directions in Relevant Logic, pages 205-225. Kluwer Academic Publishers, Dordrecht, 1989.

[9] G. fischer Servi. "On Modal Logics with an Intuitionistic Base". Studia Logica, 36:14I-I49, 1977 .

[io] James garson. "Quantification in Modal Logic". In dov m. GabBay AND FRANZ GÜNTHNER, editors, Handbook of Philosophical Logic, volume 2. Reidel, I984.

[II] JEAN-YVes Gi RARD. "Linear Logic". Theoretical Computer Science, 50:I-IOI, I987. Available online: http://iml.univ-mrs.fr/ girard/linear.pdf

[i2] G. HUghes And m. Cresswell. A Ner Introduction to Modal Logic. Routledge, London, 1996.

[i3] bernard linsky and edward n. Zalta. "In Defense of the Simplest Quantified Modal Logic". Philosophical Perspectives, 8:43I-458, 1994. 
[I4] C. RaU szer. "A Formalisation of the Propositional Calculus of H-B Logic". Studia Logica, 33:23-34, 1974.

[15] C. RaU szer. "Semi-Boolean Algebras and their Applications to Intuitionistic Logic with Dual Operations”. Fundamenta Mathematicae, 83:219-249, 1974.

[I6] GREg RESTA LL. An Introduction to Substructural Logics. Routledge, 2000.

[I7] Richard routley. "Problems and Solutions in the Semantics of Quantified Relevant Logics - I". In A. I. ARRDUdA, R. CHUAQUi, AND N. C. A. DA COSTA, editors, Proceedings of the Fourth Latin American Symposium on Mathematical Logic, pages 305-340. North Holland, I980.

[I8] Richard ROUtLey aND ROBERT K. MEyer. "Semantics of Entailment". In HUGU ES LEblanc, editor, Truth Syntax and Modality, pages 194-243. North Holland, 1973. Proceedings of the Temple University Conference on Alternative Semantics.

[I9] alex simpson. The Proof Theory and Semantics of Intuitionistic Modal Logic. PhD thesis, University of Edinburgh, 1994. available at http://homepages.inf.ed.ac.uk/als/Research/. 
The Australasian fournal of Logic (ISSN I448-5052) disseminates articles that significantly advance the study of logic, in its mathematical, philosophical or computational guises. The scope of the journal includes all areas of logic, both pure and applied to topics in philosophy, mathematics, computation, linguistics and the other sciences.

Articles appearing in the journal have been carefully and critically refereed under the responsibility of members of the Editorial Board. Only papers judged to be both significant and excellent are accepted for publication.

The journal is freely available at the journal website at

$$
\text { http://www.philosophy.unimelb.edu.au/ajl/ }
$$

All issues of the journal are archived electronically at the journal website.

SuBSCRIPTIONS Individuals may subscribe to the journal by sending an email, including a full name, an institutional affiliation and an email address to the managing editor at ajl-editors@unimelb.edu.au Subscribers will receive email abstracts of accepted papers to an address of their choice. For institutional subscription, please email the managing editor at ajl-editors@unimelb.edu.au.

Complete published papers may be downloaded at the journal's website at http: //www.philosophy.unimelb.edu.au/ajl/ The journal currently publishes in pdf format.

Submission The journal accepts submissions of papers electronically. To submit an article for publication, send the $\mathrm{LT}_{\mathrm{E}} \mathrm{X}$ source of a submission to a member of the editorial board. For a current list of the editorial board, consult the website.

The copyright of each article remains with the author or authors of that article. 